\title{
Generalized Movement Representation in Haptic Perception
}

\author{
Lucile Dupin \\ CNRS and Université Paris Descartes
}

\author{
Vincent Hayward \\ Université Pierre et Marie Curie
}

\author{
Mark Wexler \\ CNRS and Université Paris Descartes
}

\begin{abstract}
The extraction of spatial information by touch often involves exploratory movements, with tactile and kinesthetic signals combined to construct a spatial haptic percept. However, the body has many tactile sensory surfaces that can move independently, giving rise to the source binding problem: when there are multiple tactile signals originating from sensory surfaces with multiple movements, are the tactile and kinesthetic signals bound to one another? We studied haptic signal combination by applying the tactile signal to a stationary fingertip while another body part (the other hand or a foot) or a visual target moves, and using a task that can only be done if the tactile and kinesthetic signals are combined. We found that both direction and speed of movement transfer across limbs, but only direction transfers between visual target motion and the tactile signal. In control experiments, we excluded the role of explicit reasoning or knowledge of motion kinematics in this transfer. These results demonstrate the existence of 2 motion representations in the haptic system - one of direction and another of speed or amplitude - that are both source-free or unbound from their sensory surface of origin. These representations may well underlie our flexibility in haptic perception and sensorimotor control.
\end{abstract}

\begin{abstract}
Public Significance Statement
Haptic perception or active touch involves 2 types of sensory signals: tactile sensations and proprioceptive or motor signals about the movement of the fingertip or other sensory surface. While these signals usually come from the same limb, we have recently shown that tactile signals from 1 hand can be combined with proprioceptive movement information from the other hand, a phenomenon that we call "haptic transfer." Here we extend these results by showing that haptic transfer can occur between the feet and hands. Additionally, movement signals in haptic transfer can even come from eye movements or visual motion, but in this case less information is transferred than between limbs. These results indicate that, in haptic perception, information about the movement of the sensory surface is represented in a simplified way, without reference to the actual body part involved. Additionally, our results indicate that signals about movement direction might be represented differently than those about speed.
\end{abstract}

Keywords: touch, haptic perception, sensorimotor integration, kinesthesis, smooth pursuit

While the role of action in active touch has been studied for a long time (Gibson, 1962; Lederman \& Klatzky, 1987), many aspects of the conversion of proximal tactile sensations into distal and spatial haptic representations are still not well understood. During a typical movement in active touch, we accumulate a continuous stream of tactile

This article was published Online First January 12, 2017.

Lucile Dupin, Laboratoire Psychologie de la Perception, CNRS and Université Paris Descartes; Vincent Hayward, Institut des Systèmes Intelligents et de Robotique, Sorbonne Universités, Université Pierre et Marie Curie; Mark Wexler, Laboratoire Psychologie de la Perception, CNRS and Université Paris Descartes.

Correspondence concerning this article should be addressed to Lucile Dupin, Laboratoire Psychologie de la Perception, CNRS and Université Paris Descartes, 45 rue des Saints-Pères, 75006 Paris, France. E-mail: lucile.dupin@ parisdescartes.fr sensations. On one hand, the brain has access to the somatotopic location of the stimulated skin surface, its position with respect to the body. On the other hand, it has access to the spatiotopic position and movement of the sensory surface, its position or motion in space. These two information streams are combined in a way that allows us to perceive objects in space through touch.

This combination of tactile and kinesthetic signals leads to the transformation between different reference frames. The optimal reference frame depends on the task at hand. For example, to identify an object one must combine the successively touched parts into a coherent whole, and it has been shown that exploratory movement sequences play an essential role in this process (Valenza et al., 2001). To reach an object, one must locate it in egocentric space. To identify the relative locations of multiple objects requires a nonegocentric reference frame. The use of different reference frames in haptic perception has been observed: body- and object-centered (Klatzky, 1999), egocentric and allo- 
centric (Kappers, 2004; Millar \& Al-Attar, 2004 for a review) or hand-centered (Kappers \& Viergever, 2006) and allocentric (Volcic \& Kappers, 2008).

The inputs to the process of generating spatial haptic perception are tactile afferents and the kinesthetic signals concerning the position and movement of the tactile sensory surfaces. The initial reference frame of tactile signals is somatotopic. The kinesthetic signals originate from the proprioceptive system: joints, muscles, or skin mechanoreceptors (Edin \& Abbs, 1991; Edin \& Johansson, 1995; McCloskey, 1978; Newton, 1982); from motor signals in the case of active motion (Smith, Chapman, Donati, Fortier-Poisson, \& Hayward, 2009); and from tactile signals (Collins, Refshauge, Todd, \& Gandevia, 2005; Edin \& Abbs, 1991; Edin \& Johansson, 1995; McCloskey, 1978; Newton, 1982); and from signals in other modalities, if available (Lécuyer, 2009; Lécuyer, Coquillart, Kheddar, Richard, \& Coiffet, 2000). Studies of kinesthetic perception have shown the existence of biases in the estimation of distances in tactile-kinesthetic tasks such as blindfolded triangle completion (Klatzky, 1999). The geometry of perceived haptic space can be distorted by temporal factors (Dupin, Hayward, \& Wexler, 2015; Lederman, Klatzky, Collins, \& Wardell, 1987; Yusoh, Nomura, Sakamoto, \& Iwabu, 2012), movement speed (Kazunori, Akinori, Daisuke, \& Ito, 2006; Viviani, Baud-Bovy, \& Redolfi, 1997; Wapner, Weinberg, Glick, \& Rand, 1967; Whitsel et al., 1986), memory (Chieffi, Conson, \& Carlomagno, 2004; Gentaz \& Hatwell, 1999; Millar \& Al-Attar, 2004) and the configuration of the body, notably the hands (Kaas \& Mier, 2006) or the head and body (Luyat, Gentaz, Corte, \& Guerraz, 2001). Biases in the perception of orientation have been observed when participants have to orient a bar in order to make it parallel in threedimensional space to another bar (Kappers, 1999; Kappers \& Koenderink, 1999).
Another possible source of kinesthetic information is the efferent copy of the motor command that is known to play a role in vision (Bridgeman, 1995; Crapse \& Sommer, 2008; McCloskey, 1981; Wexler, 2003) and in haptic perception (Smith et al., 2009; Weiss \& Flanders, 2011).

The multiple sources of information and these different resulting reference frames in haptic spatial perception seem to imply different representations of movement. We have recently developed a method that allows us to study the coupling of tactile stimuli with movement signals in haptic perception (Dupin et al., 2015). The basic stimuli consisted of an expanding or contracting tactile line on a fingertip in perpendicular motion (see Figure 1). This ambiguous stimulus could be perceived proximally-as an expansion or contraction. Alternatively, it could be perceived as an extended distal object-a triangle felt through a slit, analogously to anorthoscopic perception in vision (Rock, 1997). We have found that most observers readily perceive the distal triangle (Dupin et al., 2015). We varied both the orientation and size (height) of the simulated triangle, as well as the direction and speed of hand motion, and had participants report both triangle orientation and size. As shown in the truth table in Figure 1C, the orientation depends both on the contraction/expansion variable and on the direction of motion, and has zero correlation with each of these individual variables-and can therefore be reported above chance level only if the two signals are combined. All participants had performance above chance, showing that the two signals are indeed combined when each one is individually insufficient to perform above chance (Dupin et al., 2015). Size judgments depended both on the size of the simulated triangle and on the duration of the tactile stimulus (Dupin et al., 2015).

Importantly, our technique allows us to "dissect" the haptic signals into separate movement and tactile streams by having one
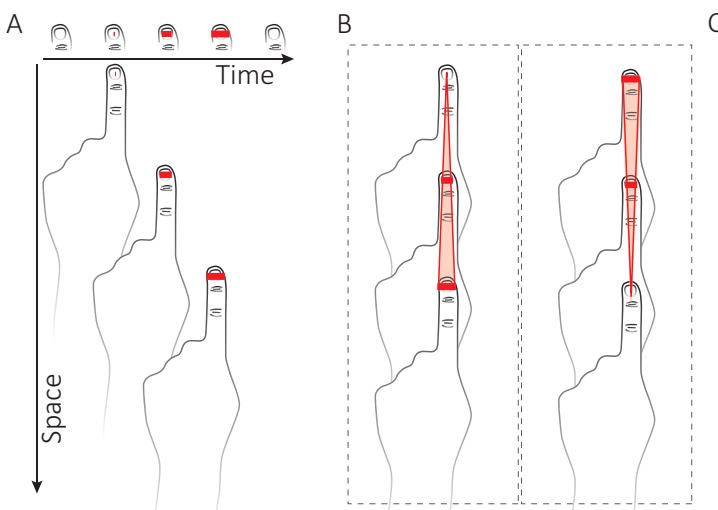

C


Figure 1. Example of one trial in normal condition for a backward movement (Space axis) and an expanding line under the finger (Time axis; A). The perceived orientation of the triangle depends on the direction of the movement and the stimulation (B). On the left, perceived triangle orientation for the combination of a forward movement and a contracting line or the combination of for a backward movement and expanding line. On the right, the perceived triangle for the combination of a forward movement and an expanding line or the combination of a backward movement and contracting line. Truth table of triangle orientations as a function of the movement direction (forward or backward) and the tactile stimulus (expansion or contraction) (C). Illustration of the link between the visual moving target and the tactile stimulation (D). The level of expansion or contraction of the tactile line depends on the location of the target on the screen. See the online article for the color version of this figure. 
hand move while the other hand remains immobile and receives the tactile consequences of the first hand's movement. This ambiguous stimulus can be interpreted in at least three different ways. If the motion and tactile signals on different hands aren't combined, then the tactile signal should be perceived as what it is: an expansion or contraction. Because this is uncorrelated with simulated triangle orientation, then performance on the orientation task would be at chance level. If the two signals are combined across hands, then there are two possibilities: direct or indirect coupling. With direct coupling, the motion signal from the moving hand is directly transferred to the hand receiving the tactile stimulus; for triangle orientation, this should lead to the same responses as in Figure 1C. With indirect coupling, the haptic system could assume that the moving hand is dragging the triangle underneath the feeling hand; in this case, the relative motion between hand and triangle should be reversed, leading to responses opposite to those in Figure 1C,

When we performed this "dissection" experiment, we found strong evidence for coupling of tactile and kinesthetic signals across hands (Dupin et al., 2015). Moreover, the coupling was direct, as if the immobile hand receiving the tactile stimuli were assumed to move in the same direction as the moving hand. Size judgments depended on the speed of the moving hand, showing that not only direction but also speed information is transferred between hands. Finally, we verified that the coupling occurs perceptually rather than on a decision level by introducing a small temporal delay between the motion and tactile stimulus, which abolished the coupling. Taken together, these results indicate that haptic perception combines tactile signals from one hand with a representation of self-motion from the other. We call this phenomenon haptic transfer.

Here we study the generality of haptic transfer. Can motion information transfer to the unmoving hand from a moving foot, the moving eyes, or visually perceived motion? Different kinds of motor coupling are known to exist between the hands and the feet (Carson, Goodman, Kelso, \& Elliott, 1995; Cavallari, Cerri, \& Baldissera, 2001), with stronger coupling between ipsilateral than contralateral members (Nakagawa, Muraoka, \& Kanosue, 2015). Motor coupling is also known to exist between the eyes and the hand (Gauthier \& Hofferer, 1976; Nishitani, Uutela, Shibasaki, \& Hari, 1999), but it is not known whether such coupling has consequences for haptic perception as well.

In Experiment 1, we measure haptic transfer between the feet and the hand, and compare it with hand-hand transfer. In Experiment 2, we check whether smooth-pursuit eye movement information and visually perceived motion transfer to the unmoving hand. In Experiment 3, we use temporal delay to study whether haptic transfer occurs perceptually or cognitively. In Experiment 4, we study whether preliminary information about motion influences haptic transfer. Finally, in Experiment 5, we test whether the haptic transfer could be caused by small involuntary movements of the feeling limb.

\section{Experiment 1}

\section{Method}

In this experiment, we compared the normal conditions of haptic perception, in which the same hand that explores an object by touch receives the tactile feedback from its movements, with three conditions requiring transfer between limbs: between the two hands (as studied previously by Dupin et al., 2015), and between a hand and ipsi- and contra-lateral feet.

Participants. Fourteen volunteers, seven males, mean age: 24 ( $S D$ 3.5), participated in this experiment and were compensated $10 €$ per hour. Two were self-declared as left-handed. All were never about the hypotheses of the study and had never participated in haptic experiments.

Apparatus. In order produce tactile stimulation on the fingertip, we used a Latero Tactile Display (Tactile Labs, Canada; Wang \& Hayward, 2009). This display has an area of $1.2 \mathrm{~cm}^{2}$ and consists of $8 \times 8$ pins that can be moved independently in one direction on the display surface (thus compressing or stretching the skin), with maximum amplitude of about $0.1 \mathrm{~mm}$ and bandwidth of about $100 \mathrm{~Hz}$.

Participants' movement was monitored using a 23-cm-long lowfriction linear slider. The slider was equipped with an optical coder connected to a dedicated electronic counter, which allowed us to retrieve the position of the platform with a precision of better than $0.1 \mathrm{~mm}$ and negligible latency. In the two foot conditions, a small skateboard (approximately $25 \times 25 \mathrm{~cm}$ ) was attached to the slider. In this configuration, the slider was positioned under the skateboard and was activated by the movement of one of the feet positioned on the skateboard. A keypad and a computer monitor were used for entering responses.

Stimuli. On each trial, the tactile stimulus was an expanding or contracting bar perpendicular to the main finger axis. This bar was displayed using some or all of the pins from two lines of the tactile display, vibrating at $70 \mathrm{~Hz}$ (the pins in the other lines remained still). The bar's length depended on the position of the slider, in order to simulate a virtual isosceles triangle felt through a slit (Figure 1A and 1B). The distance of the slider movement between the beginning and the end of the tactile stimulation is the triangle's size. There were 4 sizes: $4,8,12$, or $16 \mathrm{~cm}$. The triangle could have one of two orientations; the orientation, together with the direction of hand movement, determined whether the proximal tactile stimulus was an expansion or contraction (Figure 1C). The virtual triangle was centered on the slider with a random jitter between -1 and $+1 \mathrm{~cm}$ from trial to trial (to decrease the reliability of absolute hand position cues in size judgments). Triangle sizes and orientations were chosen randomly and independently of the direction of the participant's movement on a given trial.

Procedure. There were four conditions, each performed in a separate, single block. In all conditions, the left index fingertip was positioned on the tactile display as illustrated in Figure 2A. In the normal condition - which was always performed as the first blockthe tactile display was positioned on the slider. The left hand moved the slider while feeling the tactile stimulus. The three remaining conditions were presented in random order. In hand-contra, footcontra, and foot-ipsi conditions conditions, the movement was carried out respectively by the right hand, right foot, and left foot, while the left index fingertip was positioned on the tactile display which remained stationary (as in the normal condition). In the hand-contra condition, the right hand was positioned $40 \mathrm{~cm}$ to the right of the left hand (see Figure 2A). In the foot-contra condition, the center of the slider was aligned in the horizontal plane to the position of the right hand in hand-contra condition. In the foot-ipsi condition, the center of the slider was aligned on the left hand positioned on the tactile 
A. EXPERIMENT 1
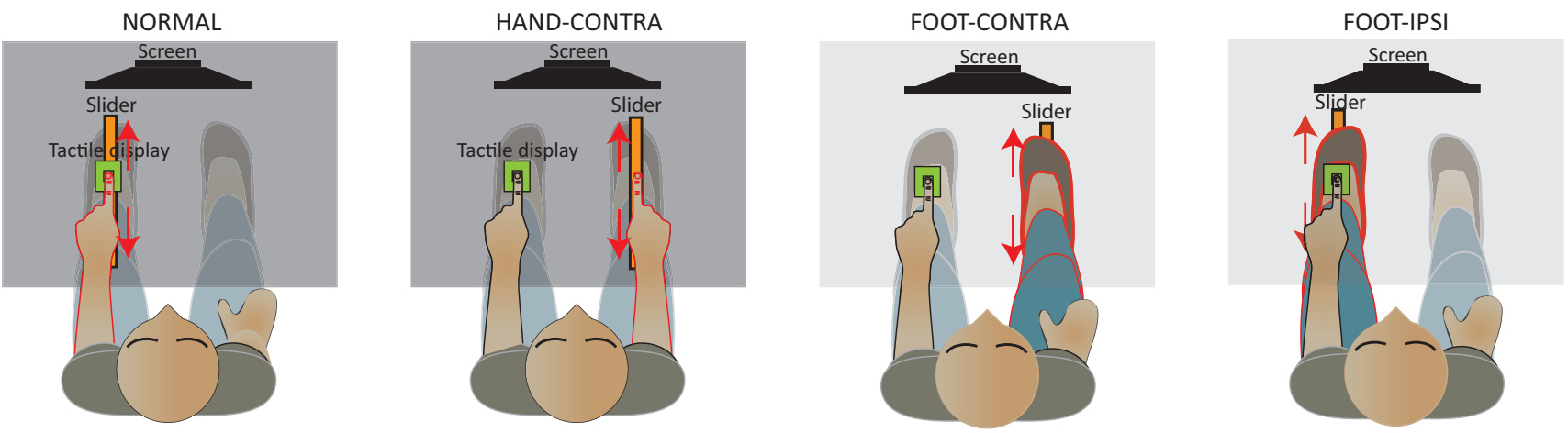

B.

EXPERIMENTS 2, 3 and 4

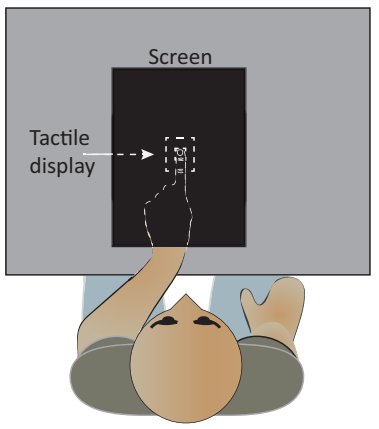

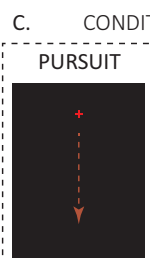

$\mathrm{OR}$

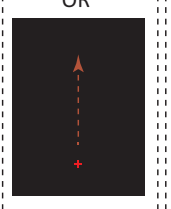

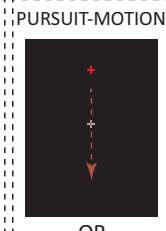

$O R$

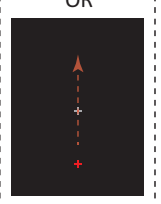

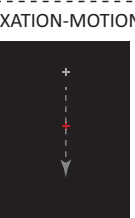

$\mathrm{OR}$

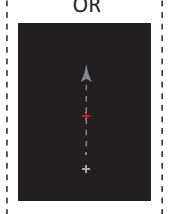

E. CONDITIONS OF EXPERIMENT 3 F. CONDITIONS OF EXPERIMENT 4 FIXTION-ASYNC

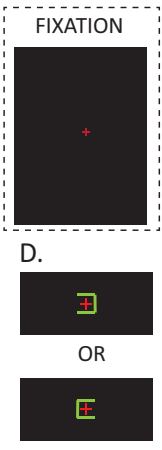

FIXATION-ASYNC

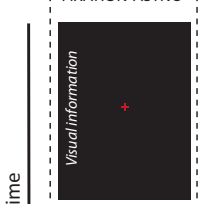

$\stackrel{\oplus}{\underline{E}}$

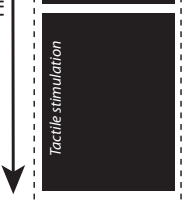

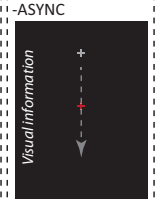
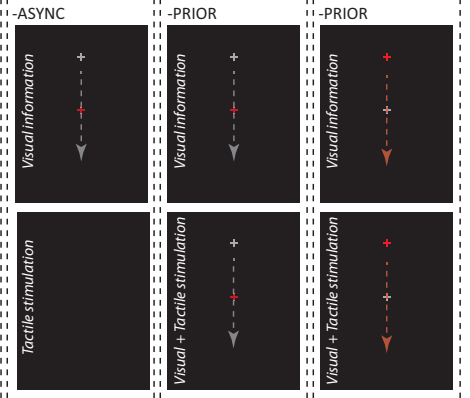

Figure 2. Illustration of the four conditions of Experiments 1 (A). Apparatus for Experiment 2, 3, and 4 (B). The transparency of the screen is only to allow seeing the hand position under the screen positioned horizontally. Illustration of the different conditions for Experiment 2 (C). Green shapes that the participant had to identify during the trial (D). Conditions of Experiment 3 (E). Only backward movement is illustrated. Conditions of Experiment 4 (F). Only backward movement is illustrated. See the online article for the color version of this figure.

display. Movement directions (backward/forward) and distances in all conditions were the same in the horizontal plane.

On each trial in all four conditions, the participant performed a continuous forward or backward movement over the full length of the slider, with the eyes closed. The tactile stimulus, as described above, was displayed during part of the movement. Participants were instructed to keep movement speed as constant as possible within each trial. In order to sample movement speeds within the range 4 to $10 \mathrm{~cm} / \mathrm{s}$ as evenly as possible, the participant was instructed to go slightly faster on each subsequent trial until reaching the speed of $10 \mathrm{~cm} / \mathrm{s}$ on a particular trial; then the experimental program instructed the participant to go slightly slower on each subsequent trial until reaching $4 \mathrm{~cm} / \mathrm{s}$, and so on. This speed instruction was given using an auditory tone after each movement, with a high (low) tone instructing the participant to move faster (slow down). After hearing the tone, the participant opened his or her eyes and reported the size and the orientation of the perceived triangle by adjusting a triangle displayed on the monitor using two keys of the keypad. A block ended when the participant performed 40 trials in the $4-10 \mathrm{~cm} / \mathrm{s}$ speed range.

The experiment began with a short presentation and demonstration of the task. Because the goal of the experiment was to test the combination of tactile and kinesthetic signals across different hands and feet, we first made sure that the signals were combined correctly on the same hand. We therefore administered a pretest, consisting of 10 random triangles in the normal condition. In order to pass the pretest, participants had to correctly report at least 8 out of 10 triangle orientations. (If participants guessed, the probability of attaining this threshold was about $5 \%$, as given by the binomial distribution.) If they did not pass the pretest on the first try, they were given it again. Eight participants passed the pretest on the first try, 3 on the second try, 2 at the third, and 1 on the fourth.

The entire experiment lasted between 60 and $90 \mathrm{~min}$.

\section{Results}

We first analyze the reported triangle direction. In the normal condition, we will express this as a fraction of correct responses, that is, ones compatible with the orientation of the simulated triangle. ${ }^{1}$ In the other conditions, we will consider responses "correct" when they are compatible with direct (rather than indirect) transfer, as discussed

\footnotetext{
${ }^{1}$ Strictly speaking, these responses are "correct" assuming that the triangle is stationary. If the triangle were to move in the same direction as the participant's hand or foot, but faster, then the "correct" response would be reversed. The assumption of stationarity is well documented both in vision (Wexler, Panerai, Lamouret, \& Droulez, 2001) and touch (RoblesDe-La-Torre \& Hayward, 2001).
} 
in the Introduction. Thus, a fraction of correct responses above 0.5 indicates direct transfer, while below 0.5 indicates indirect transfer. The results are shown in Figure 3A. The mean fractions for normal, hand-contra, foot-contra, and foot-ipsi conditions over participants were 0.83 [SD 0.13], 0.70 [0.17], 0.71 [0.13], and 0.71 [0.14], respectively. (Here and elsewhere, between-participants standard deviations will be given in square brackets.) These means were significantly above chance level, 0.5 , in all conditions, as revealed by a $t$ test (normal: $t_{13}=9.23, p<.00001$; hand-contra: $t_{13}=4.62, p<.0005$; foot-contra: $t_{13}=6.18, p<.0001$; foot-ipsi: $\left.t_{13}=5.51, p<.0001\right){ }^{2}$ The fraction of correct responses was significantly greater in the normal condition than in each of the other conditions (hand-contra: $t_{13}=2.4, p<.05$; foot-contra: $t_{13}=3.26, p<.01$; and foot-ipsi: $\left.t_{13}=3.49, p<.01\right)$. In contrast, the mean fractions did not differ significantly between the hand-contra, foot-contra, and hand-contra conditions.

Judgments of triangle size varied tremendously from one participant to the next in overall scale. This is illustrated in Figure 4A for the hand-contra condition, showing the absolute reported size as a function of simulated size, for all participants. As can be seen, the overall scale of reported sizes differed by as much as a factor of 5 to 10 between participants; however, within each participant's data, there is an orderly growth of reported size as a function of simulated size. It should also be noted that reported size is smaller than simulated size (with one exception), echoing the compression found in visual anorthoscopic (viewed through a slit) vision (Rock, 1997). In order to make different participants' data comparable, we standardized both the displayed and reported size variables for all further analysis, by converting them to $z$ scores (subtracting the mean and dividing by the standard deviation, calculated separately for each participant and condition).

We found that perceived size depended not only on simulated size, but also on stimulus duration (the time during which the participant felt the tactile stimulus on each trial). This is illustrated in Figure 4B, which shows the perceived size as a function of simulated size in the hand-contra condition (the two variables have now been standardized), separately for slow, medium, and fast speeds. Faster speeds lead to smaller perceived objects, and vice versa for slower speeds. Because movement speed and object size were varied independently, these two variables were only weakly correlated in the final data. Therefore, the effect of speed can also be seen as an effect of duration. This effect-slower speeds or longer durations leading to larger perceived objects in haptic perception-has been documented in the past (Dupin et al., 2015; Lederman \& Klatzky, 1987; Lederman, Klatzky, \& Barber, 1985).

We have therefore performed a multiple linear regression of the perceived triangle size as a function of displayed triangle size and of the stimulation duration. In order to compare the influence of size to that of duration on size perception, we also converted the durations to $z$ scores, as we had done for the sizes. The results of this regression analysis are shown graphically in Figures 5A and 6A. The mean coefficients of simulated size were 0.39 [0.17] for normal condition, 0.30 [0.24] for hand-contra, 0.37 [0.17] for foot-contra, and 0.42 [0.21] for foot-ipsi. After the fitting the linear model on individual data, we performed a second level of analysis on the coefficients obtained in the individual fits. Mean coefficients in all conditions differed significantly from 0 , as revealed by $t$ tests over participants' coefficients (normal: $t_{13}=8.52, p<$ .00001 ; hand-contra: $t_{13}=4.71, p<.0005$; foot-contra: $t_{13}=$
7.83, $p<.00001$; foot-ipsi: $\left.t_{13}=7.40, p<.00001\right)$ and did not differ significantly from one other (maximum $t_{13,2}=1.67, p>$ $.12)$.

The linear regression yielded mean coefficients of stimulation duration of 0.35 [0.24] for normal condition, 0.47 [0.24] for hand-contra, 0.37 [0.17] for foot-contra, and 0.31 [0.24] for footipsi (see Figure 5A for a graphical representation). All mean duration coefficients differed significantly from 0 , as revealed by $t$ tests over participants' coefficients (normal: $t_{13}=5.41, p<$ .0005; hand-contra: $t_{13}=7.38, p<.00001$; foot-contra: $t_{13}=$ $9.28, p<.00001$; foot-ipsi: $\left.t_{13}=3.95, p<.005\right)$. There were two marginally significant differences between normal and handcontra conditions mean coefficients $\left(t_{13,2}=2.23, p=.044\right)$ and between hand-contra and foot-ipsi $\left(t_{13,2}=2.37, p=.034\right)$. None of the other pairwise comparisons between conditions yielded significant differences (maximum $t_{13,2}=1.31, p>.21$ ).

\section{Discussion}

These results show that both the direction and amplitude of movement in haptic perception can transfer to an immobile hand from the three other distal limbs. When a stationary hand feels a tactile stimulus while another hand or foot moves, the characteristics of the resulting haptic perception are very similar to the case when the same hand both feels and moves. This coupling effect is observed in both orientation and size judgments. The mean fractions of orientation judgments were significantly above chance in all four conditions, demonstrating both the coupling and the fact that coupling was direct rather than indirect. As concerns size judgments, we found that in all four conditions reported sizes depended significantly on simulated triangle size, showing that continuous metric information can also be transferred between limbs. Thus, the perceived features of the triangle felt by the unmoving hand incorporated both discrete (direction) and continuous (size) information from another limb. These results confirm those previously found between hands (Dupin et al., 2015) and extend them to the coupling between upper and lower limbs.

\section{Experiment 2}

Given the exchange of movement information across the four distal limbs that we demonstrated in Experiment 1, we wondered what other kinds of movements signals can be coupled to tactile stimuli in the construction of haptic percepts. In Experiment 2, we studied signals from smooth-pursuit eye movements and movement of a visual stimulus on the retina. Instead of a limb movement as in Experiment 1, here we coupled the tactile expansion or contraction with visual target motion without or without smoothpursuit eye movement, as illustrated in Figure 1D.

\section{Method}

Participants. Sixteen volunteers (four males, mean age: 28 years, $S D$ 7.8) participated in this experiment and were compensated $10 €$ per hour. Two were self-declared left-handed. All were

\footnotetext{
${ }^{2}$ All data was checked for normality using Pearson's $\chi^{2}$ test prior to performing $t$ tests. No significant deviations from normality were found.
} 




B. EXPERIMENT 2

GFIXATION-MOTION EPURSUIT-MOTION EPURSUIT

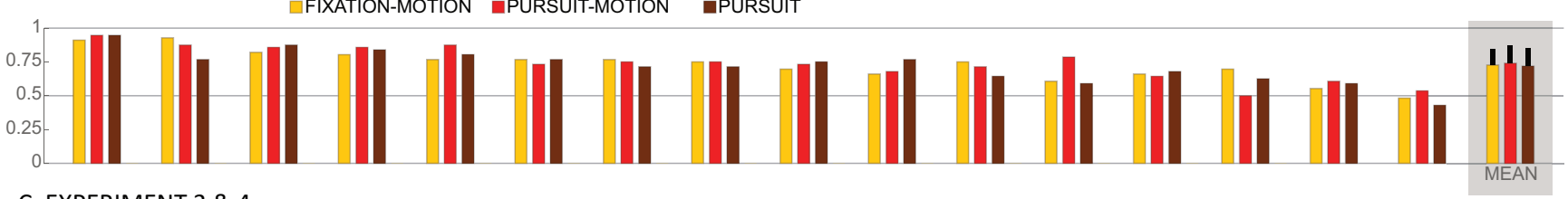

C. EXPERIMENT 3 \& 4

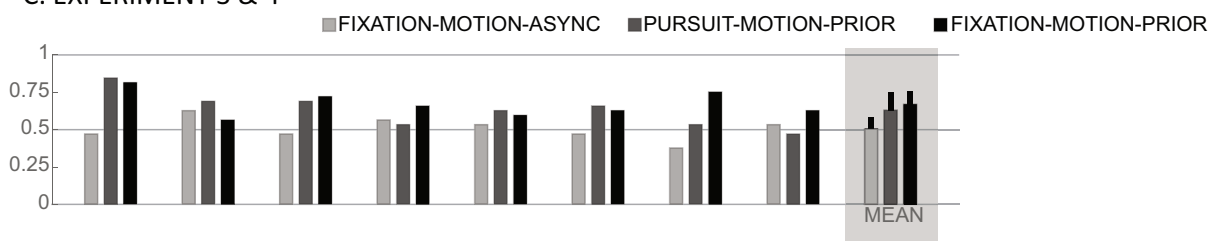

Figure 3. Ratio of triangles' orientations perceived as if it was the same hand that was moving and feeling for Experiment 1 (A), Experiment 2 (B), and Experiment 3 (C). See the online article for the color version of this figure.

naïve about the hypotheses of the study and had never participated in other haptic experiments.

Apparatus. The tactile display and the slider (for the pretest) were the same as in Experiment 1. Participants sat in front of a table, with their head movements restrained by a chinrest. The tactile display was centered in front of the participant at a distance of approximately $30 \mathrm{~cm}$ from the chinrest. A thin computer monitor (LG 15EL9500 OLED monitor, display area $33.2 \times 18.7 \mathrm{~cm}$, resolution $1,366 \times 768$, refresh rate $60 \mathrm{~Hz}$ ) was positioned horizontally over the tactile display and the hand of the participant, as shown in Figure 2B. The center of the monitor was positioned approximately $15 \mathrm{~cm}$ above the tactile display. The participant was unable to see his hand during the experiment. The distance between eyes and the center of the monitor was approximately $40 \mathrm{~cm}$.

Stimuli. The tactile stimuli were the same as in Experiment 1 (see Figure 1A, Time axis). As in Experiment 1, during the motion, the index finger of the left hand was stimulated with an expanding or contracting bar. This tactile stimulus was felt while the moving target traversed an unseen virtual triangle along its path, as shown on Figure 1D. As in Experiment 1, the triangle could have 4 different sizes $(4,8,12$, or $16 \mathrm{~cm})$ and was centered on the monitor
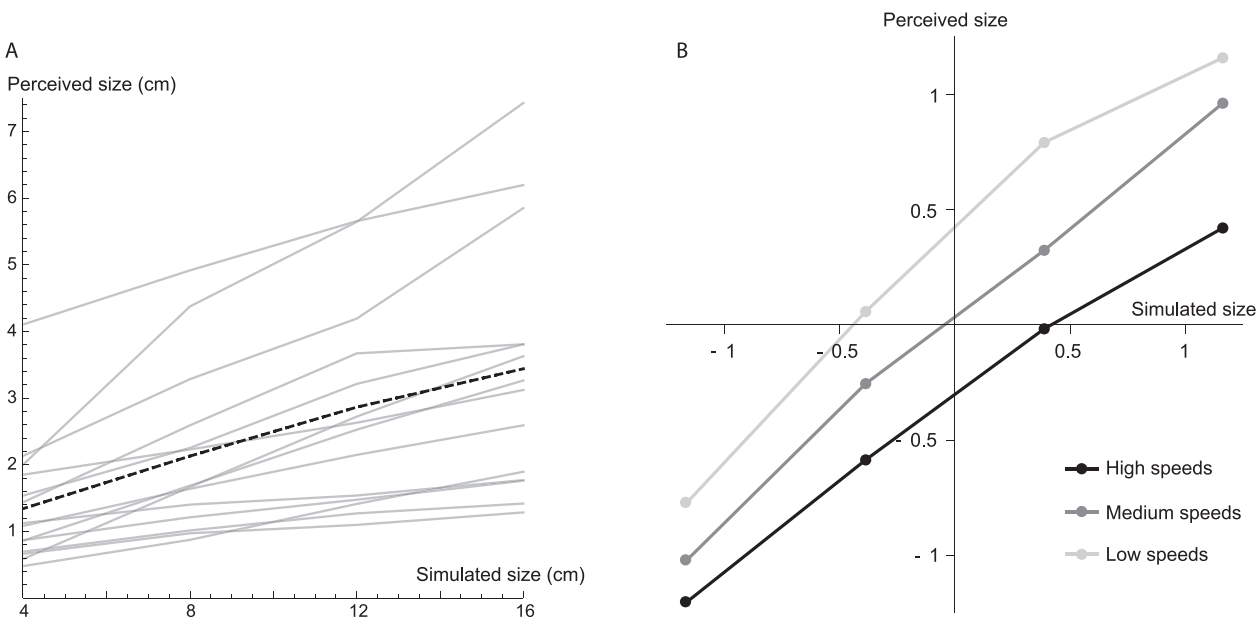

Figure 4. Mean reported size as a function of simulated size (both sizes in $\mathrm{cm}$ ), in the hand-contra condition of Experiment 1 (A). Each gray curve shows data from one participant, while the black dashed curve shows the mean. The same data, averaged over subjects, with the simulated and reported size variables converted to $z$ scores (B). The data has been divided into three terciles according to speed of movement. 

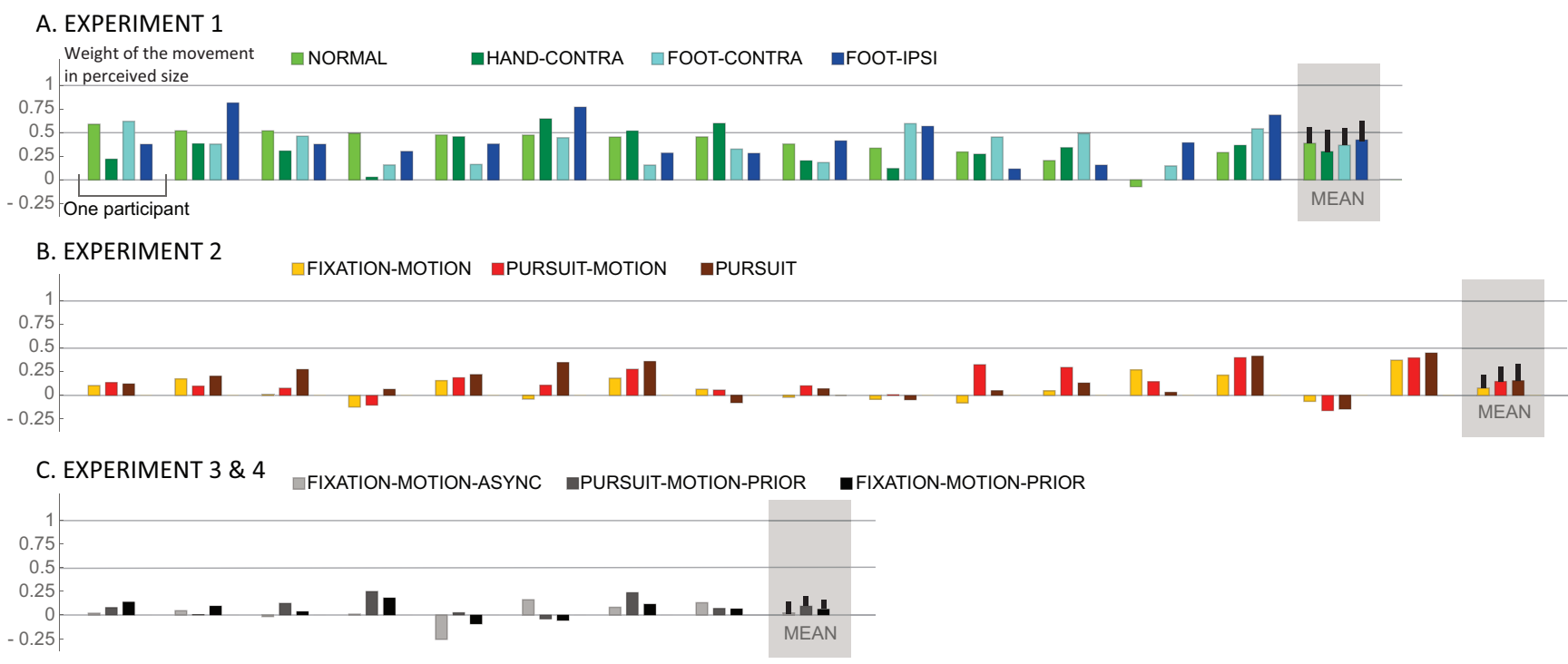

Figure 5. Fitted weight of the duration in the triangle size perceived for Experiment 1 (A), Experiment 2 (B), and Experiment 3 and $4(\mathrm{C})$, for each participant in decreasing order of mean value over all conditions. See the online article for the color version of this figure.

with a random vertical jitter between -1 and $+1 \mathrm{~cm}$. There were two possible visual stimuli that could either be displayed separately or in combination. Both stimuli were crosses (size $3.2 \mathrm{~mm}$, line width $0.24 \mathrm{~mm}$ ), and one was moving while the other was stationary. The moving cross had constant vertical speeds between 1.1 and $32 \mathrm{~cm} / \mathrm{s}$, with a total path length of $22 \mathrm{~cm}$. The stationary cross centered on the monitor. The color of each cross was determined by the task: the cross that had to be fixated during the trial was red and the other cross, if present, was gray. Another set of stimuli was used in a secondary task to control fixation and pursuit, described below.

Procedure. The four experimental conditions are illustrated in Figure $2 \mathrm{C}$. In the pursuit and pursuit-motion conditions, the participant was instructed to pursue the target that moved vertically across the monitor. In the pursuit condition, there was no other stimulus displayed on the monitor, while in the pursuit-motion condition an additional stationary cross was displayed at the center of the monitor. In fixation and fixation-motion conditions, the participant was instructed to fixate a stationary cross centered on the monitor. In the fixation condition, there was no other visual stimulus, while in the fixation-motion condition an additional cross moved vertically across the monitor. Thus, motion information, as either retinal motion or an oculomotor signal or both, was present in all conditions except fixation, which served as a control.

In the pursuit, pursuit-motion and fixation-motion conditions, while the moving stimulus traversed a virtual triangle (unseen by the participants), the left index finger received a tactile stimulus consisting of an expanding or contracting bar as in Experiment 1, corresponding to the width of the virtual triangle at the current position of the moving stimulus, as illustrated in Figures 1D and $2 \mathrm{C}$. In the fixation condition, no moving visual stimulus was displayed, but the time course of the tactile stimulus was identical to that in fixation-motion trials. The sizes of the virtual triangles were $4,8,12$, or $16 \mathrm{~cm}$, as in Experiment 1 . Visual target speeds were constant through a given trial, and were such that the target traversed the virtual triangle in $0.5,1,1.5,2,2.5,3$, or $3.5 \mathrm{~s}$ (yielding speeds between 1.1 and $32 \mathrm{~cm} / \mathrm{s}$ ). The experiment was performed in a randomized factorial design (4 conditions $\times 4$ triangle sizes $\times 7$ speeds $\times 2$ triangle orientations $=224$ trials , performed in a single block that lasted about $1 \mathrm{hr}$.

Before beginning the experiment proper, we made sure that participants could perform the triangle orientation discrimination task in the most basic condition with manual movement, with the same hand moving and feeling the tactile stimulus (equivalent to the normal condition of Experiment 1). This pretest was identical to the one in Experiment 1, but the number of trials raised to 20 with the threshold remaining at $80 \%$ to pass (expected false positive rate $0.6 \%$ ). Eleven participants passed the test on the first attempt, 4 on the second, and 1 on the third.

To ensure that participants pursued the moving target (pursuit, pursuit-motion) or fixated the immobile target (fixation, fixationmotion), there was a secondary oculomotor control task using Landolt-like stimuli, illustrated in Figure 2D, consisting of a square with either the left or right side missing. The square was displayed centered on the moving or stationary cross fixated or pursued by the participant. The square was green, and its size was $5 \mathrm{~mm}$ (line width $0.24 \mathrm{~mm}$ ). One to eight such squares were displayed during each trial, with each presentation lasting $80 \mathrm{~ms}$, during motion (or virtual motion in fixation condition) randomly chosen among the eight $2-\mathrm{cm}$ segments of the 16 central $\mathrm{cm}$ of the motion path. The secondary task was to report the orientation of the final square. The task was impossible to do outside the fovea due to crowding, and the target appeared too briefly to execute a saccade.

At the start of each trial, the fixation or pursuit target (red cross) appeared. In the two fixation conditions, it was centered on the monitor (directly above the left index fingertip resting on the tactile display underneath). In the two pursuit conditions, it ap- 
peared at the top or the bottom of the monitor, depending on its subsequent motion direction (Figure 2C). The participant was instructed to fixate the target and to start the trial by pressing a key. In the fixation conditions, the target remained in the same position throughout the trial (the participant was instructed to fixate it), while in the pursuit conditions, the target moved at constant speed to the opposite edge of the monitor, and the participant was instructed to pursue it. At the end of the trial, he or she reported the orientation and size of the perceived triangle by adjusting a triangle displayed on the monitor using two keys of the keypad, as in Experiment 1. Following this response, the participant reported the orientation of the final oculomotor control shape.

\section{Results}

The fraction of correct responses on the oculomotor control task by participant varied between $75 \%$ and $99 \%$ (mean $88 \%, S D$ $6.9 \%)$.

As in Experiment 1, we calculated the fraction of trials in which the direction of motion was combined with the tactile stimulus in accordance with the truth table of Figure 1C. In the two pursuit conditions, the motion direction was that of the pursuit target; in fixation-motion, it is the direction of the visual motion. Individual results and group means are shown in Figure 3B for the three conditions with motion. The mean fractions were 0.72 [0.13] for pursuit, 0.74 [0.13] for pursuit-motion, and 0.73 [0.12] for fixation-motion. These means were significantly above chance level, 0.5 , in all conditions, as revealed by $t$ tests over participants' means (fixation-motion: $t_{15}=7.65, p<.00001$; pursuit-motion: $t_{15}=7.59, p<.00001$; pursuit: $\left.t_{15}=6.91, p<.00001\right)$. There were no significant differences between any of the conditions: fixation-motion and pursuit-motion (maximum $t_{15,2}=1.1, p=$ $.30)$. Because there was no motion signal in the fixation condition, orientation responses could not be analyzed in the same way.
When comparing the fraction of correct responses between Experiment 1 and 2, the only significant difference was between normal (mean 0.83) and pursuit conditions $\left(t_{28}=2.33, p=.03\right)$ and between normal and fixation-motion conditions $\left(t_{28}=2.24\right.$, $p<.03)$. All other paired comparisons were not significantly different $\left(t_{28}<1.89, p>.07\right)$.

In the three conditions with motion information, we performed a linear regression of perceived triangle versus real size (amplitude of movement during the stimulation) and stimulus duration, after converting all variables to $z$ scores. Individual and group means are shown in Figure 5B. The mean coefficient of size was 0.15 [0.18] for pursuit, 0.17 [0.16] for pursuit-motion, and 0.08 [0.14] for fixation-motion. The mean coefficients in pursuit and pursuitmotion conditions differed significantly from 0 , as revealed by $t$ tests over participants' means $\left(t_{15}=3.43, p<.01\right.$, and $t_{15}=3.61$, $p<.01$, respectively), whereas fixation-motion only did so marginally $\left(t_{15}=2.18, p=.045\right)$. Pairwise comparisons between conditions showed that the differences between the three conditions did not attain significance (maximum $t_{15,2}=2.07, p=.056$ ).

We now turn to the coefficients of duration in the linear regressions. In the fixation condition, where participants had no access to a motion signal and therefore had no independent information about size, the linear regression was of perceived size versus stimulus duration, the only available variable. The individual and mean coefficients of duration are shown in Figure 6B. Mean duration coefficients were 0.58 [0.17] for pursuit, 0.58 [0.14] for pursuit-motion, 0.58 [0.11] for fixation-motion, and 0.68 [0.08] for fixation. In all conditions, the means differed significantly from 0 (pursuit: $t_{15}=16.4$; pursuit-motion: $t_{15}=13.9$; fixation-motion: $t_{15}=20.7$; fixation: $t_{15}=33.2$, all have $p<.0001$ ). Pairwise comparisons between conditions revealed no significant differences (maximum $t_{15,2}=0.07, p=.94$ ).

Comparing these results to corresponding analyses in Experiment 1 , we have found on one hand that size coefficients in all
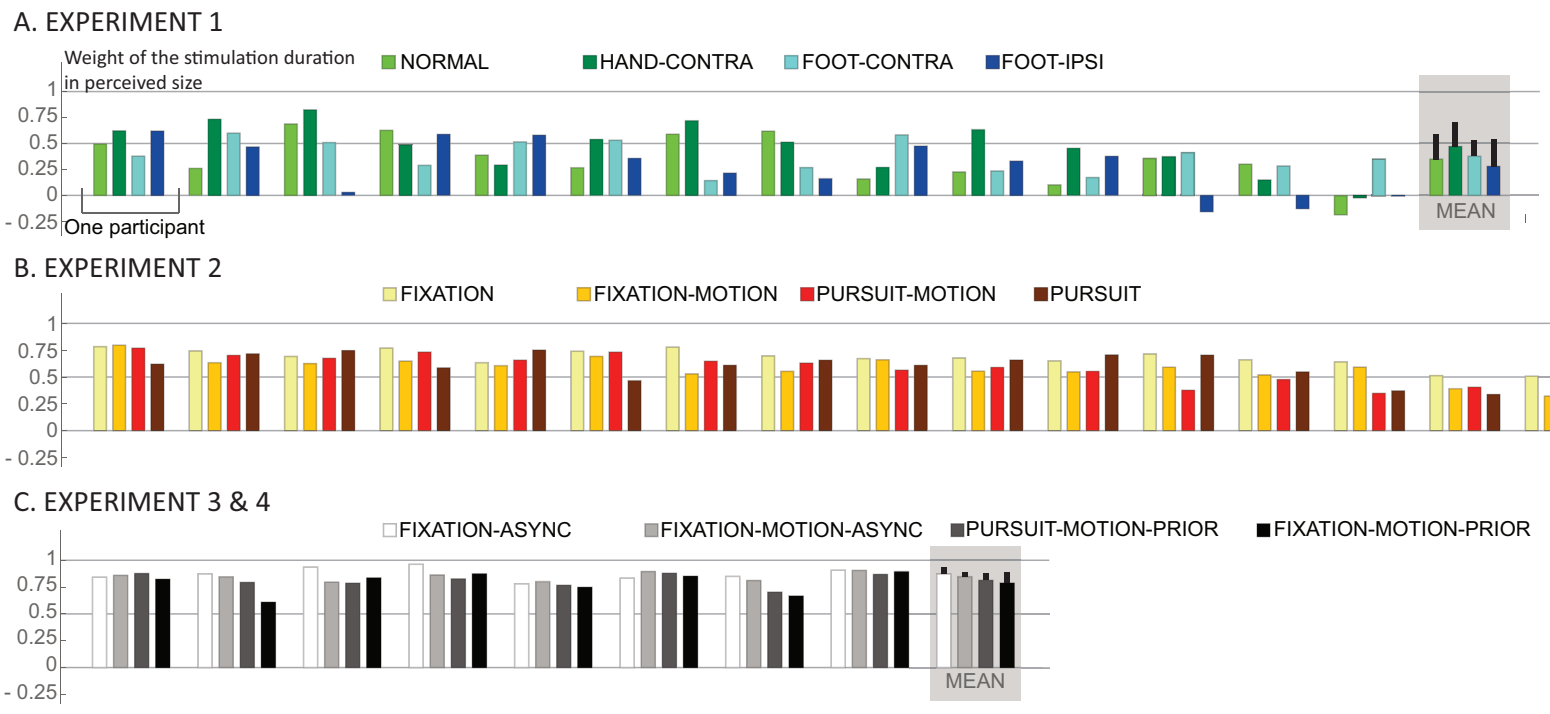

Figure 6. Fitted weight of movement in triangle perceived size for Experiment 1 (A), Experiment 2 (B), and Experiment 3 and 4(C), for each participant sorted ordered as in Figure 5. See the online article for the color version of this figure. 
conditions of Experiment 2 were significantly lower than all their counterparts in Experiment 1: 0.39 for normal condition $\left(t_{28}>3.6\right.$, $p<.005), 0.30$ for hand-contra $\left(t_{28}>2.1, p<.05\right), 0.37$ for foot-contra $\left(t_{28}>3.3, p<.005\right)$, and 0.42 foot-ipsi $\left(t_{28}>3.7, p<\right.$ .001) conditions - with the exception of the comparison between pursuit and hand-contra conditions $\left(t_{28}=1.9, p=.07\right)$. On the other hand, the duration coefficients in Experiment 2 were significantly higher than their counterparts in the normal (0.35), foot-ipsi (0.31), and foot-contra (0.37) conditions of Experiment $1\left(t_{28}>\right.$ $3.05, p<.01)$. The hand-contra duration coefficient from Experiment 1 was different from fixation $\left(t_{28}=3.1, p<.01\right)$ conditions but not from pursuit, pursuit-motion, and fixation-motion conditions $\left(t_{28}<1.6, p>.14\right)$.

\section{Discussion}

The results show that, in the majority of trials, triangle orientation is perceived in accordance with the rule illustrated in Figure 1B. The tactile sensation on the immobile finger is associated with the movement direction, which is sourced either from the direction of eye movement (in the pursuit-motion and pursuit conditions) or from retinal motion (in the fixation-motion condition). In the pursuit-motion condition, these two directions are in conflict: when the eyes move upward or away from the participant in a spatiotopic reference frame, the stationary cross moves downward in retinotopic coordinates or toward the participant. Our results show that the eye movement direction, or motion of the pursuit in spatiotopic coordinates, is the one that is integrated with the tactile sensation resulting in the haptic perception.

As in Experiment 1, we have calculated how the perceived size of the triangle depended on the spatial extent of the visual target's motion during the tactile stimulation, and on the duration of the tactile stimulation. We have found in all conditions that the perceived size depended strongly on the duration. But the use of spatial extent was weak: the size coefficients were lower than in Experiment 1, whereas the duration coefficients were higher. Indeed, perceived size in this experiment mostly depended on the duration of the tactile stimulation, rather than on the spatial extent of the target motion. Even though the target's motion direction was taken into account in the perception of the triangle's orientation, the spatial extent of the target's motion contributed little to the perception of the triangle's size, in contrast to both the hand and foot conditions of Experiment 1. These results raise the possibility of two distinct representations, one for the direction and the other one for the spatial extent of the participant's movement.

\section{Experiment 3}

We have assumed that, when participants in Experiments 1 and 2 reported triangle orientations, they were reporting their perceptions, arrived at through an implicit and unconscious application of the truth table in Figure 1C. However, it is also possible that participants were explicitly and consciously reasoning about the geometry of the stimulus and deducing the same responses. In order to check if our participants' performance was due to perception or to deduction, we performed a new experiment in which we applied a small temporal delay (mean duration $604 \mathrm{~ms}$ ) between the motion and the tactile stimulus. Such delays have been found to impede perceptual integration (Barrouillet, Bernardin, Portrat,
Vergauwe, \& Camos, 2007), but should not hamper explicit reasoning (Cowan, Saults, \& Nugent, 1997; Lewandowsky, Duncan, \& Brown, 2004). We have performed a similar control experiment in our recent study (Dupin et al., 2015) on hand-to-hand transfer (conditions similar to the normal and hand-contra conditions of Experiment 1), and found that asynchronies between the two signals strongly impeded performance. In a subsequent block, we explicitly taught the rule in Figure $1 \mathrm{C}$ to our participants and had them repeat the experiment, this time deliberately applying the learned rule. In this condition, performance was once again high, showing that the temporal delay itself did not impede reasoning. Taken together, these results strongly supported unconscious perceptual integration of movement and tactile signals, rather than conscious deduction of the responses. In this experiment, we applied asynchronies to a subset of the conditions of Experiment 2 , in order to test whether the integration of visual and tactile signals was due to perception or deduction.

\section{Method}

Participants. Eight volunteers (3 males, mean age 25, SD 4.5 years) participated in this experiment. They were compensated $10 €$ per hour. All were self-declared right-handed. All were naïve concerning the hypotheses of the study, and 7 had never participated in experiments on haptic perception.

Apparatus and stimuli. The apparatus and stimuli are the same as in Experiment 2, apart from changes in timing that are described below.

Conditions. There were two conditions: fixation-motionasync and fixation-async, similar to the fixation-motion and fixation conditions of Experiment 2, except that the visual and tactile stimuli were not synchronized, but rather the visual stimulus preceded the tactile stimulus (see Figure 2E). Each trial was the temporal juxtaposition of the visual and tactile parts of a trial in Experiment 2.

At the start of a trial, a red fixation cross appeared at the center of the monitor, as in Experiment 2. The participant was instructed to fixate the cross and start the trial by pressing a key. In fixationmotion-async, a gray cross moved across the monitor, as in fixation-motion of Experiment 2. Fixation-async, with no movement information, served as a control condition. After a temporal delay, which depended on the size of the triangle and the duration of the visual stimulus (see below), the tactile stimulus was displayed. Following the tactile stimulation, there was a delay before the participant could respond, equal to the delay between the visual and the tactile stimuli.

There were two triangle orientations, two triangle sizes (12 and $16 \mathrm{~cm}$ ) and 4 stimulation durations: $0.5,1.5,2.5$, and $3.5 \mathrm{~s}$. The experiment was performed in a randomized factorial design with each condition performed once, yielding 64 trials. The experiment lasted approximately $15 \mathrm{~min}$.

Because a trial was the temporal of the visual and tactile components of an Experiment 2 trial, there was a temporal interval between the offset of the visual stimulus and the onset of the tactile stimulus. This was because the tactile stimulus did not begin at once, but rather when the moving target (here seen only in the first half of the trial) reached the virtual triangle, located a few centimeters from the start of the $22 \mathrm{~cm}$ path (see Figure 1D). For example, if the triangle was $12 \mathrm{~cm}$ long and centered on the 
monitor, the target would have had to travel $(22-12) / 2 \mathrm{~cm}$ before reaching the triangle. If the stimulus duration in this trial was 1.5 seconds, the target speed would have been $12 / 1.5=8 \mathrm{~cm} / \mathrm{s}$, and so $5 \mathrm{~cm}$ would have taken $0.625 \mathrm{~s}$ to travel. Thus, on such a trial, there would have been a $0.625 \mathrm{~s}$ interval between the offset of the visual stimulus and the onset of the tactile stimulus. The mean interstimulus interval was $604 \mathrm{~ms}$ (SD 457, minimum $94 \mathrm{~ms}$, maximum $1,458 \mathrm{~ms}$ ).

Procedure and pretest. The pretest and the experiment were identical to the pretest of Experiment 2. Five participants passed the test on the first attempt, and 3 on the second.

\section{Results}

As in previous experiments, we have analyzed (see Figure 3C) the fraction of responses in fixation-motion-async following the rule illustrated on Figure 1C. The fixation-async condition was excluded from this analysis because there was no movement in this condition. The ratio of fixation-motion-async responses was 0.50 [0.08] and not significantly different from the 0.5 chance level $\left(t_{7}=0.14, p=.23\right)$ but significantly different from the fixationmotion condition of Experiment $2\left(t_{22}=4.83, p<.0001\right)$. In a multiple regression of reported sizes versus real size and stimulus duration, the size coefficient (Figure 5C) was 0.02 [0.13] for fixation-motion-async and was not significantly different from 0 $\left(t_{7}=0.48, p=.65\right)$. The duration coefficients (Figure 6C) were 0.87 [0.06] in fixation-motion-async and 0.85 [0.04] in fixationasync and were not different from each other $\left(t_{7}=1.11, p=.30\right)$ but different from 0 (respectively $t_{7}=41.6, p<.0001 ; t_{7}=57.7$, $p<.0001)$.

When comparing these coefficients to those of Experiment 2, we have found that size coefficients did not differ significantly between fixation-motion-async and fixation-motion conditions $\left(t_{22}=1.89, p=.07\right)$. Duration coefficients were significantly greater in fixation-motion-async condition than in fixation-motion condition $\left(t_{22}=6.94, p<.00001\right)$ and in fixation-async condition than in fixation condition $\left(t_{22}=5.42, p<.0005\right)$.

\section{Discussion}

In the fixation-motion-async condition, the reported triangle orientations were no different from chance, meaning that the direction of motion of the visual target was unlikely to have been taken into account in any systematic way, and in particular following the truth table in Figure 1C. In addition, the weight of the spatial extent of the visual target's motion in size judgments was not significantly different from 0 , meaning that the target motion's metric features were also not used in haptic size judgments. Indeed, the only stimulus dimension that predicted size judgments was temporal duration, and the dependence on duration in the fixation-motion-async condition was no less than in fixationasync, in contrast to the results obtained in Experiment 2.

These results show that when the visual movement and the tactile stimulation were not synchronized (separated by a small temporal interval), there was no cognitive or perceptual combination of the visual and tactile stimuli, insofar as neither the direction nor the amplitude of visual target motion was taken into account in reported triangle orientation or size. The small asynchrony of the visual and the tactile stimuli should block their unconscious or implicit combination in perception, but should still allow their conscious or explicit combination through deductive reasoning. The lack of any combination in the asynchronous condition, in contrast to the equivalent synchronous condition of Experiment 2, supports the notion that the signals were combined through perception rather than explicit reasoning in the synchronous conditions of the previous experiments.

\section{Experiment 4}

We have observed different results in Experiments 1 and 2 concerning the effect on size judgments of the metric spatial information provided by the movement. In Experiment 1, the metric spatial information from hand or foot movement (its amplitude or speed) clearly played a role in perceived triangle size, as evidenced by significantly positive coefficients of the size parameter in the linear regression of reported size versus size and duration. In contrast, in Experiment 2, the metric spatial information from visual target motion (either retinal motion, or oculomotor signals accompanying smooth pursuit) played a much smaller role in size judgments, which were almost entirely based on stimulus duration. One difference between these two experiments was that in Experiment 1, the participant had prior information about movement speed because he or she initiated this movement following explicit speed instructions, to move faster or slower than the preceding trial. In Experiment 2, the speed of the visual target on a given trial became available only after the trial began. The aim of Experiment 4 was to check whether this difference of results between Experiments 1 and 2 was due to the prior information about movement speed. To do so, we have added to two conditions of Experiment 2 a prior presentation of the visual target motion before each trial. The two conditions in this experiment were pursuit-motion-prior and fixation-motion-prior, corresponding respectively to pursuit-motion and fixation-motion of Experiment 2 to which was added a prior presentation of target motion was added. The results of this experiment were compared with those of Experiment 2 in order to identify a potential role of the prior knowledge about the movement.

\section{Method}

Participants. The 8 participants of Experiment 3 took part in Experiment 4, which was carried out immediately following Experiment 3 .

Apparatus and stimuli. The apparatus and stimuli were the same as those of Experiment 2, apart from the additional presentations of target motion that are described below.

Conditions. The two conditions were pursuit-motion-prior and fixation-motion-prior. These conditions were similar to the pursuit-motion and fixation-motion conditions, respectively, from Experiment 2 except that the visual stimulus was displayed twice during each trial: once without the tactile stimulus, and then immediately a second time together with and synchronized to the tactile stimulus, exactly as in Experiment 2 (see Figure 2F). The two presentations of the visual stimulus were identical to one another.

The trial began with the onset of the red stationary fixation cross (as in Experiments 2 and 3). The participant was instructed to fixate the cross and start the trial by pressing a key. In fixation- 
motion-prior condition, the fixation cross remained in the center of the monitor while a gray cross moved across the monitor. The opposite motions took place in the pursuit-motion-prior condition: the red cross, which the participant was instructed to pursue, moved across the monitor, while a gray cross remained stationary in the center (see conditions of Experiment 2 and Figure 6F). The participant had to either fixate or pursue the red cross at all times. The red cross then disappeared and immediately reappeared at its initial position and from that point the trial is identical to the equivalent condition in Experiment 2.

As in Experiment 3, there were two triangle orientations, two triangle sizes (12 and $16 \mathrm{~cm}$ ) and 4 stimulation durations: 0.5, 1.5, 2.5 , and $3.5 \mathrm{~s}$. The experiment was performed in a randomized factorial design with each condition performed once, yielding 64 trials. The experiment lasted approximately $15 \mathrm{~min}$.

\section{Results}

The fraction of perceived triangle orientations taking into account the direction of the movement (as illustrated in Figure 1C) was 0.63 [0.12] in the pursuit-motion-prior condition and 0.67 [0.09] in fixation-motion-prior (Figure 3C). Both were significantly different from chance level 0.5 (respectively $t_{7}=3.07, p=$ .02 , and $\left.t_{7}=5.58, p<.01\right)$. The triangle orientation results in the two conditions were not significantly different $\left(t_{7,2}=0.95, p=\right.$ .38). They were also not significantly different from results in pursuit-motion $\left(t_{22}=2.1, p=.051\right)$ and fixation-motion $\left(t_{22}=\right.$ $1.2, p=.23$ ) conditions in Experiment 2.

As in the previous experiments, we performed a linear regression of perceived triangle versus spatial (amplitude of movement during the stimulation) temporal (stimulus duration) parameters, after converting all variables to $z$ scores. The weight of the spatial parameter (Figure 5C) for fixation-motion-prior condition was not significantly different from 0 (mean 0.06 [0.09], $t_{7}=1.78, p=$ .12). This coefficient was marginally different from 0 (mean 0.09 [0.11], $t_{7}=1.78, p=.04$ ) for pursuit-motion-prior condition. The fixation-motion-prior and pursuit-motion-prior duration weights were not significantly different from those in the fixation-motion (0.08 [0.14], $\left.t_{22}=0.32, p=.75\right)$ and pursuit-motion (0.17 [0.16], $t_{22}=0.85, p=.41$ ) conditions in Experiment 2 .

The weights of the temporal parameter in perceived size (Figure 6C) were 0.81 [0.06] in the fixation-motion-prior and 0.79 [0.10] in the pursuit-motion-prior conditions, and were not different from each other $\left(t_{7,2}=0.93, p=.38\right)$. The coefficient in the fixationmotion-prior condition was significantly different from that of fixation-motion in Experiment 2 (0.58 [0.14], $t_{22}=5.48, p<$ $.0005)$ and fixation-motion-prior from fixation-motion $(0.58$ [0.11], $\left.t_{22}=3.8, p<.005\right)$.

\section{Discussion}

We have found very little effect of prior visual motion presentation. The weights of the spatial parameter in size judgments are still very close to zero, as they were in the corresponding conditions without prior presentation in Experiment 2. The weights of the temporal parameters were higher than in Experiment 2. The judgments of triangle orientation were very similar to those in Experiment 2.

We wondered whether prior knowledge of motion speed played a role in the difference between the results of Experiments 1 and
2, namely that in Experiment 1, where there was prior information about speed, and the spatial parameter played an important role in size judgments; whereas in Experiment 2, where no prior information was available about speed, the spatial parameter played little or no role in size judgments. If so, we should have found, on one hand, an improvement in the weight of the spatial parameter, and on the other hand, a decrease of the weight of the temporal parameter in this experiment. We have found neither effect, and indeed, we have found an increase in the weight of the temporal parameter as compared with Experiment 2.

Taken together, these results imply that the absence or near absence of the use of the metric spatial parameter (motion extent or speed, in contrast to motion direction) is not due to the absence of prior knowledge about motion speed, whether for smooth pursuit or retinal motion.

\section{Experiment 5}

In talking about haptic transfer, we have assumed that it takes place covertly, through neural channels: a representation of the movement of the moving limb, or the moving eye, or the visual motion, is somehow applied to the hand that receives the tactile input. However, in at least some cases of transfer, the coupling could, in principle, be through overt movement. For example, consider the hand-contra condition of Experiment 1, in which one hand moves, while the other hand is supposed to remain still on the tactile display. Because of bimanual motor coordination, the feeling hand could actually execute small movements in the same direction as the moving hand, and these small, involuntary movements could combine with the tactile inputs from the same hand to generate the perception of triangle orientation. This explanation is less plausible as an account of size perception (because any involuntary movements would be too small in amplitude or speed), or in the case of oculomotor- or visual-to-haptic transfer, but we wanted to rule it out in the case of hand-to-hand transfer. We therefore repeated the hand-contra condition of Experiment 1, measuring any displacement of the hand on the tactile display.

\section{Method}

Unless stated, all methods were identical to the hand-contra condition of Experiment 1. In this condition, the right hand moved the slider, while the index finger of the left hand rested on the tactile display. We measured any spontaneous movements of the hand on the tactile display by filming the trials using a video camera placed overhead (Sony Handycam HDR-CX305; 1,440 $\times$ 1,080 resolution, 25 frames/s). We placed a small sticker on the back of this hand in order to facilitate the coding of its position. We first localized the first and last frames of the movement of the right hand. We then coded the displacement of the left hand between two moments in each trial: the first frame of the right hand's movement, and at $2 / 3$ of the duration of the movement. (We chose the $2 / 3$ point in order to avoid contamination by any possibly backward movement at the moment the other hand stops.)

Four participants took part in the experiment ( 2 men, mean age 23). As in Experiment 1, each participant completed a pretest, which consisted of 20 random stimuli in the normal condition. In order to pass the pretest, participants had to correctly report triangle orientation on at least $80 \%$ out of 20 
trials. Three participants passed the pretest on the first try, while one passed it on the second try.

The main block consisted of 40 trials. The conditions were identical to those of the hand-contra block of Experiment 1, except that the triangles only had sizes of 8 and $16 \mathrm{~cm}$, and that participants did not receive any instructions as to the speed of their movements.

\section{Results}

We analyzed the directions of the small, occasional spontaneous movements of the hand that received the tactile stimuli and that was supposed to remain immobile. Forward movements were coded as +1 , backward movements as -1 , and trials with no detectable movement were coded as $0.40 \%$ of the trials had detectable movement. We found that the mean movement measure when the other hand moved forward was -0.01 , and when the other hand moved backward it was -0.06 , averaged over participants. A sign test revealed no statistical differences between these means $(p>.6)$.

\section{Discussion}

This experiment tested the hypothesis that transfer of motion representations between hands is mediated by bimanual motor coordination producing spontaneous overt movements in the nominally still hand in the same direction as the moving hand. We tracked the direction of any spontaneous movements by the nominally still hand that received the tactile stimuli. We found no significant evidence for these spontaneous movements being preferentially in the direction of the other hand's movement. Thus, there is no evidence for movement transfer being mediated by overt bimanual coordination.

\section{General Discussion}

In this study, we used shape and size perception tasks to probe the combination of tactile signals on an immobile hand with kinesthetic movement signals from another member, or a motion signal from the eyes in smooth pursuit, or motion on the retina. Observers could only report shape (triangle orientation) above chance level if they combined the direction of movement with the direction of expansion or contraction of the tactile stimulus. Reported size provided another test of haptic signal combination, in which timing information from the tactile signal had to be combined with information about movement amplitude or speed for the perceived triangle size to reflect simulated size.

In the first experiment, we found that a foot movement can be coupled with the tactile stimulation located on a distal and immobile hand. The direction of foot movement is coupled with the tactile sensation of the hand in the perception of the triangle's shape. The perceived size is a combination between the duration of the stimulation and an estimation of the movement during this stimulation (Dupin et al., 2015). There were few differences in the coupling of signals across different members, whether they came from the opposite hand, the same (ipsilateral) foot, or the opposite foot. These results show that haptic perception uses an abstract representation of movement independent of the limb that moves (and of the corresponding muscles and joints).
Effector-independent movement coding has been observed in the case of transfer learning experiments (Grafton, Hazeltine, \& Ivry, 1998; Swinnen et al., 2010; van Mier \& Petersen, 2006). Our results show that an effector-independent representation is likewise used in haptic perception.

In the second experiment, we have tested if this abstract representation of movement extends to visual motion of a target that is either tracked by the eyes or moves on the retina. Although haptic perception is often accompanied by corresponding visual stimuli, and kinesthetic and visual motion are sometimes coupled (Gauthier \& Hofferer, 1976; Steinbach \& Held, 1968), visual motion is obviously less tightly linked to tactile sensations than are kinesthetic signals, which always accompany active touch. The results showed that two aspects of movement couple differently with the tactile signal on an immobile hand. One feature of the movement, its direction, couples with the tactile signal to an extent comparable to hand and foot movement as found in Experiment 1-as shown by reports of triangle orientation. The second feature, information about the spatial extent or speed of the movement, hardly at all couples to the tactile signal, as opposed to hand and foot movement - as shown by reports of triangle size.

The aim of the third experiment was to examine if the coupling of movement direction and tactile sensation observed in the second experiment was due to a perceptual or a cognitive process. To do so, we have added a small delay between the movement direction information and the tactile stimulation. The delay should inhibit perceptual but not a cognitive integration (Barrouillet et al., 2007; Cowan et al., 1997). In this case, the orientation reported by participants was independent of the movement direction, meaning that there was no coupling of the movement and tactile signals. This shows that the results of Experiment 2 were due to a perceptual process, rather than a conscious deduction. The difference found in Experiment 2 between the integration of motion but no integration of amplitude or extent could have been explained if observers used a cognitive process, and assuming that direction is cognitively easier to integrate with the tactile signal (after all, there are only four possibilities-see Figure 1C) than the continuous variable of spatial extent. However, we have shown in Experiment 3 that cognitive or deductive processes do not account even for the direction integration in Experiment 2. Therefore, we can conclude that the pattern found in Experiment 2-integration of the discrete variable of direction and the nonintegration of the continuous variable of extent-is a truly perceptual effect.

In Experiment 4, we explored a difference between Experiments 1 and 2 that could have led to different results that we have found concerning the integration of spatial extent or speed, namely integration when another limb moved (Experiment 1) and the absence of integration in the case of eye motion or motion on the retina (Experiment 2). In Experiment 1, the instruction about the target movement speed before each trial gave observers prior information that was not available in Experiment 2, where motion speed was completely unpredictable. This difference in predictability could have led to the difference in the results we have found concerning the integration of spatial extent or speed. In Experiment 4 , we modified the procedure of Experiment 2 by adding a prior presentation of the movement before each trial. We have found that this prior knowledge did not improve the integration of spatial extent, excluding that the differences about the integration 
of spatial extent between the first and the second experiment were due to the prior knowledge about movement.

In a previous study (Dupin et al., 2015), we have shown that the movement of one hand can be associated with a tactile stimulation located on the other hand that is immobile. The spatial perception arising from this association was as if the immobile hand receiving the tactile stimulus was moving in roughly the same way-in the same direction and at the same speed-as the mobile hand. But the link between the hands is special due to bimanual coordination, and could have explained the association we had observed.

In the four studies described here, we have found a common representation for the movement direction of the hand, foot, eyes, or retinal motion abstracted from where it originates over the body. This representation was associated with a tactile stimulus located on an immobile hand in order to generate the perception of a triangle oriented in space as if the immobile hand moved in the same direction as the moving hand, foot, or visual target. We have also found transfer of the metric characteristics (speed or amplitude) of the movement - the perceived size of the triangle reflected the speed or extent of the movement - in the case of limb (hand or foot) movement, but for visual target motion.

Thus, we have found evidence of two abstract or sourceindependent representations of movement in the haptic system, one of direction and one of speed or amplitude. The representation of movement is a complex problem for the haptic system, because of the large number of potential sensory surfaces that are at most weakly coupled biomechanically, thus leading to a highdimensional space of possible movements, as well as to a binding problem of connecting each movement to its sensory surface of origin, which we will call its source. Our results show that the haptic system does not represent each movement in strict association with its source. Instead, its representation of movement is simplified. It could represent movements, but without binding them to their sources. In our case, this would mean that the haptic system "knows" there's a limb moving forward and another remaining still, but does not know whether the limb moving forward is the same as the one receiving the tactile input or not. Alternatively, the representation of movement by the haptic system could have a very narrow "bandwidth," being limited to the representation of at most one movement. Future studies will have to distinguish between these two types of representations.

The difference between the representation of the direction and the amplitude or speed of a movement has been previously observed in studies of the variabilities of these two components for reaching movements (Gordon, Ghilardi, \& Ghez, 1994) or, in the context of spatial cognition, in path completion (Klatzky, 1999). There were several differences between limb and visual target motion that could explain why the metric characteristics of the movement (speed or amplitude) were transferred in the case of limb movement but not for visual target motion. One difference was the prior planning and knowledge of the movement in the case of limb movement, and its absence in the other conditions. Experiment 4 has excluded this possible explanation. A second difference is that, in the limb conditions, the movement was actively generated by the participant whereas in visual target motion, the task was either to pursue the target, or in the retinal motion condition, to perform no movement at all. In these two later conditions, there was either no active control of the motion trajectory (pursuit) or no motor action at all. Active motor control is known to improve accuracy in target tracking (Steinbach, 1969), haptic perception (Smith et al., 2009), and anticipation of the position of a moving target (Wexler \& Klam, 2001). A third and obvious difference is that in the visual target condition, the movement transfer would have to be cross-modal, originating from visual or oculomotor signals. Although touch and vision that are known to interact in the case of spatial attention (Martino \& Marks, 2000; Spence, Pavani, \& Driver, 2000), visual perception (Ernst, Banks, \& Bülthoff, 2000; Lunghi \& Alais, 2013; Lunghi, Binda, \& Morrone, 2010; Pettypiece, Goodale, \& Culham, 2010), or tactile perception (Pettypiece et al., 2010), their coupling may be more limited than that between tactile and kinesthetic signals.

More generally, the loss of source information about the origin of movement can be seen as a limitation-only one movement can be represented at a time-or as a simplification - the source information could be ignored because another sensory-motor coupling criterion, for example temporal synchrony, is more important than any initial binding to the source sensory surface. In order to check the generality of the haptic transfer phenomenon, it would be interesting, for example, to study the situation in which the two limbs move simultaneously in different directions and to measure the effect on haptic perception in one limb of movement in the other. Finally, the source-free representation of motion allows flexibility and adaptability of the sensory-motor associations, as can be observed in many ordinary situations such as tool use (Iriki, Tanaka, \& Iwamura, 1996; Yamamoto \& Kitazawa, 2001), the use of simple "teleoperation" devices such computer mice and joysticks, and in the use of visuo-tactile sensory substitution systems (Bach-y-Rita, Collins, Saunders, White, \& Scadden, 1969; Bachy-Rita \& Kercel, 2003).

\section{References}

Bach-y-Rita, P., Collins, C. C., Saunders, F. A., White, B., \& Scadden, L. (1969). Vision substitution by tactile image projection. Nature, 221, 963-964. http://dx.doi.org/10.1038/221963a0

Bach-y-Rita, P., \& Kercel, S. W. (2003). Sensory substitution and the human-machine interface. Trends in Cognitive Sciences, 7, 541-546. http://dx.doi.org/10.1016/j.tics.2003.10.013

Barrouillet, P., Bernardin, S., Portrat, S., Vergauwe, E., \& Camos, V. (2007). Time and cognitive load in working memory. Journal of Experimental Psychology: Learning, Memory, and Cognition, 33, 570-585. http://dx.doi.org/10.1037/0278-7393.33.3.570

Bridgeman, B. (1995). A review of the role of efference copy in sensory and oculomotor control systems. Annals of Biomedical Engineering, 23 , 409-422. http://dx.doi.org/10.1007/BF02584441

Carson, R. G., Goodman, D., Kelso, J. A. S., \& Elliott, D. (1995). Phase transitions and critical fluctuations in rhythmic coordination of ipsilateral hand and foot. Journal of Motor Behavior, 27, 211-224. http://dx .doi.org/10.1080/00222895.1995.9941711

Cavallari, P., Cerri, G., \& Baldissera, F. (2001). Coordination of coupled hand and foot movements during childhood. Experimental Brain Research, 141, 398-409. http://dx.doi.org/10.1007/s00221-001-0898-3

Chieffi, S., Conson, M., \& Carlomagno, S. (2004). Movement velocity effects on kinaesthetic localisation of spatial positions. Experimental Brain Research, 158, 421-426. http://dx.doi.org/10.1007/s00221-0041916-Z

Collins, D. F., Refshauge, K. M., Todd, G., \& Gandevia, S. C. (2005). Cutaneous receptors contribute to kinesthesia at the index finger, elbow, and knee. Journal of Neurophysiology, 94, 1699-1706. http://dx.doi.org/ 10.1152/jn.00191.2005 
Cowan, N., Saults, J. S., \& Nugent, L. D. (1997). The role of absolute and relative amounts of time in forgetting within immediate memory: The case of tone-pitch comparisons. Psychonomic Bulletin \& Review, 4, 393-397. http://dx.doi.org/10.3758/BF03210799

Crapse, T. B., \& Sommer, M. A. (2008). Corollary discharge across the animal kingdom. Nature Reviews Neuroscience, 9, 587-600. http://dx .doi.org/10.1038/nrn2457

Dupin, L., Hayward, V., \& Wexler, M. (2015). Direct coupling of haptic signals between hands. Proceedings of the National Academy of Sciences of the United States of America, 112, 619-624. http://dx.doi.org/ 10.1073/pnas.1419539112

Edin, B. B., \& Abbs, J. H. (1991). Finger movement responses of cutaneous mechanoreceptors in the dorsal skin of the human hand. Journal of Neurophysiology, 65, 657-670.

Edin, B. B., \& Johansson, N. (1995). Skin strain patterns provide kinaesthetic information to the human central nervous system. The Journal of Physiology, 487, 243-251.

Ernst, M. O., Banks, M. S., \& Bülthoff, H. H. (2000). Touch can change visual slant perception. Nature Neuroscience, 3, 69-73. http://dx.doi .org/10.1038/71140

Gauthier, G. M., \& Hofferer, J. M. (1976). Eye tracking of self-moved targets in the absence of vision. Experimental Brain Research, 26, 121-139. http://dx.doi.org/10.1007/BF00238277

Gentaz, E., \& Hatwell, Y. (1999). Role of memorization conditions in the haptic processing of orientations and the "oblique effect." British Journal of Psychology, 90, 373-388. http://dx.doi.org/10.1348/ 000712699161477

Gibson, J. J. (1962). Observations on active touch. Psychological Review, 69, 477-491. http://dx.doi.org/10.1037/h0046962

Gordon, J., Ghilardi, M. F., \& Ghez, C. (1994). Accuracy of planar reaching movements. Experimental Brain Research, 99, 97-111. http:// dx.doi.org/10.1007/BF00241415

Grafton, S. T., Hazeltine, E., \& Ivry, R. B. (1998). Abstract and effectorspecific representations of motor sequences identified with PET. The Journal of Neuroscience, 18, 9420-9428.

Iriki, A., Tanaka, M., \& Iwamura, Y. (1996). Coding of modified body schema during tool use by macaque postcentral neurones. Neuroreport, 7, 2325-2330. http://dx.doi.org/10.1097/00001756-199610020-00010

Kaas, A. L., \& Mier, H. I. (2006). Haptic spatial matching in near peripersonal space. Experimental Brain Research, 170, 403-413. http:// dx.doi.org/10.1007/s00221-005-0223-7

Kappers, A. M. L. (1999). Large systematic deviations in the haptic perception of parallelity. Perception, 28, 1001-1012. http://dx.doi.org/ $10.1068 / \mathrm{p} 281001$

Kappers, A. M. L. (2004). The contributions of egocentric and allocentric reference frames in haptic spatial tasks. Acta Psychologica, 117, 333340. http://dx.doi.org/10.1016/j.actpsy.2004.08.002

Kappers, A. M. L., \& Koenderink, J. J. (1999). Haptic perception of spatial relations. Perception, 28, 781-795. http://dx.doi.org/10.1068/p2930

Kappers, A. M. L., \& Viergever, R. F. (2006). Hand orientation is insufficiently compensated for in haptic spatial perception. Experimental Brain Research, 173, 407-414. http://dx.doi.org/10.1007/s00221-0060377-y

Kazunori, T., Akinori, K., Daisuke, M., \& Ito, A. (2006). Haptic length display based on cutaneous-proprioceptive integration. Journal of Robotics and Mechatronics, 18, 489-498. http://dx.doi.org/10.20965/jrm .2006.p0489

Klatzky, R. L. (1999). Path completion after haptic exploration without vision: Implications for haptic spatial representations. Perception \& Psychophysics, 61, 220-235. http://dx.doi.org/10.3758/BF03206884

Lécuyer, A. (2009). Simulating haptic feedback using vision: A survey of research and applications of pseudo-haptic feedback. Presence, 18, 39-53. http://dx.doi.org/10.1162/pres.18.1.39
Lécuyer, A., Coquillart, S., Kheddar, A., Richard, P., \& Coiffet, P. (2000). Pseudo-haptic feedback: Can isometric input devices simulate force feedback? In Proceedings IEEE Virtual Reality 2000 (Cat. No. 00CB37048; pp. 83-90). http://dx.doi.org/10.1109/VR.2000.840369

Lederman, S. J., \& Klatzky, R. L. (1987). Hand movements: A window into haptic object recognition. Cognitive Psychology, 19, 342-368. http://dx.doi.org/10.1016/0010-0285(87)90008-9

Lederman, S. J., Klatzky, R. L., \& Barber, P. O. (1985). Spatial and movement-based heuristics for encoding pattern information through touch. Journal of Experimental Psychology: General, 114, 33-49. http:// dx.doi.org/10.1037/0096-3445.114.1.33

Lederman, S. J., Klatzky, R. L., Collins, A., \& Wardell, J. (1987). Exploring environments by hand or foot: Time-based heuristics for encoding distance in movement space. Journal of Experimental Psychology: Learning, Memory, and Cognition, 13, 606-614. http://dx.doi.org/10 $.1037 / 0278-7393.13 .4 .606$

Lewandowsky, S., Duncan, M., \& Brown, G. D. A. (2004). Time does not cause forgetting in short-term serial recall. Psychonomic Bulletin \& Review, 11, 771-790. http://dx.doi.org/10.3758/BF03196705

Lunghi, C., \& Alais, D. (2013). Touch interacts with vision during binocular rivalry with a tight orientation tuning. PLOS ONE, 8, e58754 http://dx.doi.org/10.1371/journal.pone.0058754

Lunghi, C., Binda, P., \& Morrone, M. C. (2010). Touch disambiguates rivalrous perception at early stages of visual analysis. Current Biology, 20, R143-R144. http://dx.doi.org/10.1016/j.cub.2009.12.015

Luyat, M., Gentaz, E., Corte, T. R., \& Guerraz, M. (2001). Reference frames and haptic perception of orientation: Body and head tilt effects on the oblique effect. Perception \& Psychophysics, 63, 541-554. http://dx .doi.org/10.3758/BF03194419

Martino, G., \& Marks, L. E. (2000). Cross-modal interaction between vision and touch: The role of synesthetic correspondence. Perception, 29, 745-754. http://dx.doi.org/10.1068/p2984

McCloskey, D. I. (1978). Kinesthetic sensibility. Physiological Reviews, 58, 763-820.

McCloskey, D. I. (1981). Corollary discharges: Motor commands and perception. In R. Terjung (Ed.), The nervous system, motor control. Handbook of physiology. Hoboken, NJ: Wiley.

Millar, S., \& Al-Attar, Z. (2004). External and body-centered frames of reference in spatial memory: Evidence from touch. Perception \& Psychophysics, 66, 51-59. http://dx.doi.org/10.3758/BF03194860

Nakagawa, K., Muraoka, T., \& Kanosue, K. (2015). Potential explanation of limb combination performance differences for two-limb coordination tasks. Physiological Reports, 3, e12301. http://dx.doi.org/10.14814/phy2 .12301

Newton, R. A. (1982). Joint receptor contributions to reflexive and kinesthetic responses. Physical Therapy, 62, 22-29.

Nishitani, N., Uutela, K., Shibasaki, H., \& Hari, R. (1999). Cortical visuomotor integration during eye pursuit and eye-finger pursuit. The Journal of Neuroscience, 19, 2647-2657.

Pettypiece, C. E., Goodale, M. A., \& Culham, J. C. (2010). Integration of haptic and visual size cues in perception and action revealed through cross-modal conflict. Experimental Brain Research, 201, 863-873. http://dx.doi.org/10.1007/s00221-009-2101-1

Robles-De-La-Torre, G., \& Hayward, V. (2001). Force can overcome object geometry in the perception of shape through active touch. Nature, 412, 445-448. http://dx.doi.org/10.1038/35086588

Rock, I. (1997). Anorthoscopic perception. In I. Rock (Ed.), Indirect perception (pp. 107-126). Cambridge, MA: MIT Press.

Smith, A. M., Chapman, C. E., Donati, F., Fortier-Poisson, P., \& Hayward, V. (2009). Perception of simulated local shapes using active and passive touch. Journal of Neurophysiology, 102, 3519-3529. http://dx.doi.org/ 10.1152/jn.00043.2009

Spence, C., Pavani, F., \& Driver, J. (2000). Crossmodal links between vision and touch in covert endogenous spatial attention. Journal of 
Experimental Psychology: Human Perception and Performance, 26 1298-1319. http://dx.doi.org/10.1037/0096-1523.26.4.1298

Steinbach, M. J. (1969). Eye tracking of self-moved targets: The role of efference. Journal of Experimental Psychology, 82, 366-376. http://dx .doi.org/10.1037/h0028115

Steinbach, M. J., \& Held, R. (1968). Eye tracking of observer-generated target movements. Science, 161, 187-188. http://dx.doi.org/10.1126/ science.161.3837.187

Swinnen, S. P., Vangheluwe, S., Wagemans, J., Coxon, J. P., Goble, D. J., Van Impe, A., . . . Wenderoth, N. (2010). Shared neural resources between left and right interlimb coordination skills: The neural substrate of abstract motor representations. NeuroImage, 49, 2570-2580. http:// dx.doi.org/10.1016/j.neuroimage.2009.10.052

Valenza, N., Ptak, R., Zimine, I., Badan, M., Lazeyras, F., \& Schnider, A. (2001). Dissociated active and passive tactile shape recognition: A case study of pure tactile apraxia. Brain: A Journal of Neurology, 124, 2287-2298. http://dx.doi.org/10.1093/brain/124.11.2287

van Mier, H. I., \& Petersen, S. E. (2006). Intermanual transfer effects in sequential tactuomotor learning: Evidence for effector independent coding. Neuropsychologia, 44, 939-949. http://dx.doi.org/10.1016/j .neuropsychologia.2005.08.010

Viviani, P., Baud-Bovy, G., \& Redolfi, M. (1997). Perceiving and tracking kinesthetic stimuli: Further evidence of motor-perceptual interactions. Journal of Experimental Psychology: Human Perception and Performance, 23, 1232-1252. http://dx.doi.org/10.1037/0096-1523.23.4.1232

Volcic, R., \& Kappers, A. M. L. (2008). Allocentric and egocentric reference frames in the processing of three-dimensional haptic space. Experimental Brain Research, 188, 199-213. http://dx.doi.org/10.1007/ s00221-008-1353-5

Wang, Q., \& Hayward, V. (2009). Biomechanically optimized distributed tactile transducer based on lateral skin deformation. The International Journal of Robotics Research, 29, 323-335. http://dx.doi.org/10.1177/ 0278364909345289
Wapner, S., Weinberg, J., Glick, J. A., \& Rand, G. (1967). Effect of speed of movement on tactualkinesthetic perception of extent. The American Journal of Psychology, 80, 608-613. http://dx.doi.org/10.2307/1421193

Weiss, E. J., \& Flanders, M. (2011). Somatosensory comparison during haptic tracing. Cerebral Cortex, 21, 425-434. http://dx.doi.org/10.1093/ cercor/bhq110

Wexler, M. (2003). Voluntary head movement and allocentric perception of space. Psychological Science, 14, 340-346. http://dx.doi.org/10 1111/1467-9280.14491

Wexler, M., \& Klam, F. (2001). Movement prediction and movement production. Journal of Experimental Psychology: Human Perception and Performance, 27, 48-64. http://dx.doi.org/10.1037/0096-1523.27 .1 .48

Wexler, M., Panerai, F., Lamouret, I., \& Droulez, J. (2001). Self-motion and the perception of stationary objects. Nature, 409, 85-88. http://dx doi.org/10.1038/35051081

Whitsel, B. L., Franzen, O., Dreyer, D. A., Hollins, M., Young, M., Essick, G. K., \& Wong, C. (1986). Dependence of subjective traverse length on velocity of moving tactile stimuli. Somatosensory Research, 3, 185-196. http://dx.doi.org/10.3109/07367228609144583

Yamamoto, S., \& Kitazawa, S. (2001). Sensation at the tips of invisible tools. Nature Neuroscience, 4, 979-980. http://dx.doi.org/10.1038/ nn721

Yusoh, S. M. N. S., Nomura, Y., Sakamoto, R., \& Iwabu, K. (2012). A study on the duration and speed sensibility via finger-pad cutaneous sensations. Procedia Engineering, 41, 1268-1276. http://dx.doi.org/10 .1016/j.proeng.2012.07.310

Received February 28, 2016

Revision received September 9, 2016

Accepted September 15, 2016 